\title{
Accessibility of urban spaces for visually impaired pedestrians
}

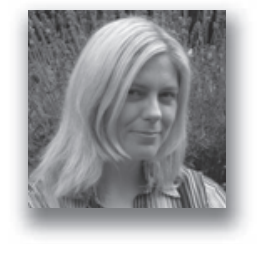

Sarah H. Norgate PhD, MBPsS, PGCert

Senior Lecturer in Psychology, University of Salford, Greater Manchester, UK

An ageing demographic together with the predicted increase in visual impairment of older people calls for a renewed consideration of the accessibility and social inclusivity of urban spaces. This paper synthesises the evidence on this topic and highlights areas for future development relating to the accessibility of urban areas for older, blind and partially sighted pedestrians in the light of recommendations and statements by the World Health Organisation's 'Age friendly' cities initiative, Guide Dogs UK and the Department of Transport's shared spaces local transport note.

\section{Introduction}

Although there is a recognised need to increase the accessibility of future urban environments for older pedestrians (e.g. World Health Organisation (Who), 2007), what remains underrepresented to date is evidence-based provision for those who are both older and blind or partially sighted. Between 2010 and 2050, there is anticipated to be a dramatic increase in age-related eye diseases with a $184 \%$ increase in age-related macular degeneration and 138\% increase in cataracts (based on UK estimates, Access Economics (2009)). Unless there is adequate planning and provision of municipal services and facilities, environmental barriers to independent and safe personal mobility will increase for these pedestrians.

Visual impairment is an established risk factor for loss of independence (Gallagher et al., 2011), being one of the four most significant potential contributors to loss of independence among older people (Alliance for Aging Research, 1999). However, loss of independence is not inevitable, and Douglas et al. (2011) emphasise the role played by rehabilitation workers to facilitate assessment and support for people who have acquired visual loss including provision of access to mobility devices (e.g. long cane, guide dog).

Within the context of equality legislation (e.g. UK Equality Act 2010 (2010)), there is a duty of care to tackle discrimination and promote equality of opportunity. Applied to the issue of accessibility of urban spaces, not only does this require safe and independent access to familiar urban centres and high streets but also, importantly, to unfamiliar spaces. In terms of the perceptions of visually impaired pedestrians, a survey by Johnson and Petrie (1998) showed that respondents (aged 2675 years) rated their satisfaction with independent travel in unfamiliar environments as low.
Notably, for any pedestrian - regardless of visual status navigating unfamiliar streets will typically incur a higher cognitive load than is the case with familiar streets. However, whereas sighted pedestrians have spontaneous access to a looming optic flow about street hazards or new landmarks, pedestrians with substantial functional vision loss rely on sequential perceptual information - particularly auditory or tactile. In this respect, depending on any mobility aids used, the cognitive load is higher owing to the sequential nature of the access. For example, an auditory stream of traffic sounds and intermittent gaps is one source of information for blind pedestrians, potentially aiding alignment to the road when walking alongside it or before considering crossing it (Wall Emerson et al., 2011). In places without designated crossings, it is the sequential detection of auditory cues (e.g. reduction of engine revs coincident with braking) which informs the timing of the decision to make a safe crossing. More needs to be understood about the cognitive constraints associated with reliance on a sequential auditory stream in urban landscapes.

Regarding access to 'safe' mobility, as far as the author is aware, data about road casualties associated with visually impaired pedestrians are not systematically collected at a national level. However, from a survey of 163 visually impaired adults, some 47 respondents reported an experience where their cane had been run over and 13 reported actually having been hit by a vehicle (Carroll and Bentzen, 1999). In addition, in terms of subjective perceptions of personal safety, people who are blind or visually impaired are known to report a fear of falling (Gallagher et al., 2011). As there is evidence of falls being causally related to blindness or partial sight, this fear is justified. A review of 31 studies by Legood et al. (2002) demonstrates that people with a sight loss are 1.7 times more likely to have a fall. In economic terms, the cost associated with falls attributable to partial sight and blindness in those 
Accessibility of urban spaces for visually impaired pedestrians Norgate aged above 60 years amounted to around $£ 20$ million of public funding in 2008 alone (Access Economics, 2009).

Recently, three separate international or national initiatives, all independently informed by their own relevant research evidence base, have chosen to put the pedestrian at the forefront of urban design. The respective positions of the Who 'Age friendly' cities initiative (Who, 2007), the Department of Transport (DfT) shared spaces local transport note (DfT, 2011) and the Guide Dogs UK 'Streets ahead' campaign (Guide Dogs UK, 2012) are now reviewed below.

The 'age-friendly cities' initiative by Who (2007) aimed to engage cities in being more inclusive for all users, especially older adults. This led to a series of recommendations of 'essential features', notably for 'outdoor spaces' and 'transportation'. By eliciting views from 1485 older adults (60-74 years; $75+$ years) and service providers from 33 cities in 22 countries regarding the features of their city they viewed as 'age friendly', a number of recommendations were made (Plouffe and Kalache, 2010). Although a minority of the initiative's recommendations did include features that emphasised the non-visual modalities (e.g. pedestrian crossings have visual and audio signals), significantly there was no reported systematic involvement with user groups who were blind or partially sighted, at least for this phase of the research.

In the second initiative, with the same intention of making the pedestrian a priority, a number of 'shared space' schemes in Europe (e.g. Netherlands, Germany) have been implemented. In the UK, there is the DfT local transport note (DfT, 2011) 'Shared space' initiative. Of particular relevance to high streets, the task was to improve pedestrian movement and comfort by reducing the dominance of motor vehicles and enabling all users to share the space rather than follow the clearly defined rules implied by more conventional designs'.

As shown in Figure 1, the tangible indicators of sharing include non-segregated space. Drivers and cyclists give way to pedestrians occupying the carriage-way. The pedestrian experience would involve crossing the street at their point of choice be it in terms of location, angle and/or time of choosing. Aside from these physical characteristics, the quality of the pedestrian experience is viewed as important. Factors like the extent to which the street would actually be experienced as a desirable place to spend time - enhanced by leisure activities, sense of place and social cohesion - were important.

One contentious issue from the shared space scheme is the extent to which surfaces are to be shared. On streets with level surfaces, pedestrians and drivers are not segregated. In terms of designing shared space, one of the requirements is that 'the scheme should be comfortable to use and accessible to disabled

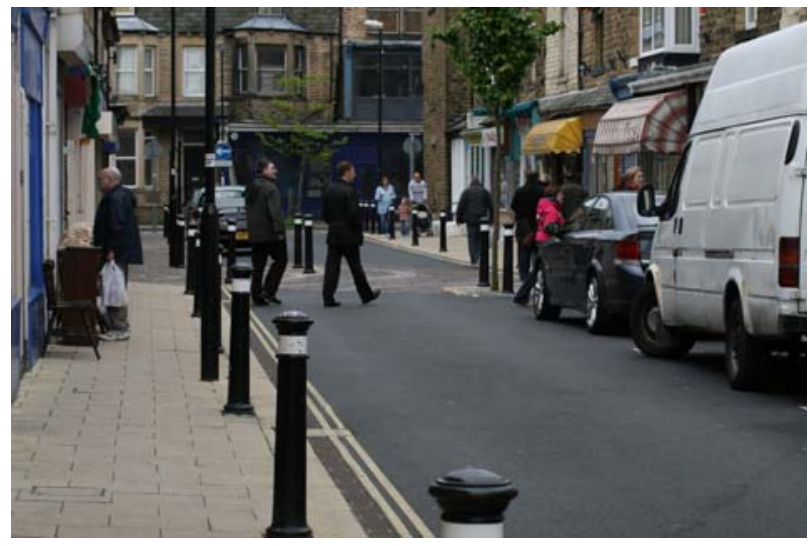

Figure 1. Photograph demonstrating how tangible indicators of sharing include non-segregated space

people' (DfT, 2011). Regarding users with visual impairment, the issues around 'level' surfaces and tactile paving can be particularly contentious, as will be discussed later.

In terms of evidence for the efficacy of the shared space schemes, whereas some studies have sought and found evidence for resultant reduction of road casualties (e.g. HamiltonBaillie, 2008), others have focused on perceptions of users towards pedestrian comfort and driver willingness to reduce road speed. A study by Kaparias et al. (2012) involved respondents putting themselves in the position of either the driver or the pedestrian. Under various hypothetical scenarios (e.g. high as opposed to low vehicle traffic; high as opposed to low pedestrian traffic; many as opposed to few children/elderly etc.), the task was to indicate whether or not as a pedestrian or a driver respondents would be comfortable using a shared space. For example, they found that when the pedestrian density is high and includes children and older pedestrians, drivers are less willing to share space. Yet conversely, for pedestrians, the same configuration is associated with pedestrian comfort. Although the scenarios in this study included vulnerable users who were children, older people and wheelchair users, notably there was no inclusion of scenarios involving pedestrians who were blind or visually impaired (either with or without various mobility aids). Further, the restriction to hypothetical written scenarios clearly lacks a degree of ecological validity and warrants future investigation in situ.

Previous survey reports by Thomas (2008) highlight the 'before and after' surveys of shared space in New Road, Brighton, UK, which aired concerns by blind and partially sighted users that shared space would reduce independence, be more difficult to navigate and negatively affect their confidence. The local council was responsive to this feedback and subsequently 
Accessibility of urban spaces for visually impaired pedestrians Norgate engaged in initiatives that sought to introduce the concept of a 'safe space' in which there was spatial segregation of vehicles from pedestrians. From a methodological perspective, alongside work which establishes user perspectives on 'shared space', there is also a niche to develop controlled research which occurs in situ, comparing measures of pedestrian mobility performance and satisfaction across a range of relevant test conditions (e.g. shared as opposed to non-shared space; familiar as opposed to unfamiliar).

The third initiative, the 'Streets ahead' campaign by Guide Dogs UK (2012) advocated the need for inclusive principles to underpin the design of both new and existing streetscapes. In addition, this was to be achieved alongside moves to equip blind and partially sighted people with the tools to engage in communication with local authorities, engineers, architects and town planners, so as to ensure consultations are effective in taking their needs into account.

In summary, all three initiatives have underpinned their position with research - either directly with participants in the case of Who (2007) or by research programmes by Guide Dogs UK and in the case of DfT 'Shared space' local transport note (DfT, 2011) through consultancy MVA (2009, 2011a, 2011b). To inform future planning and provision, it is a logical next step to assess the degree of alignment between these multiple lines of work. The key statements from Guide Dogs UK, Who (2007) and UK DfT (2011) on accessibility are next reviewed according to a number of key common themes identified from the three sets of documentation. These are 'pedestrian priority', 'walkways and pedestrian routes', 'pavements, kerbs and surface differentiation', 'street crossings and intersections' and 'access to transit systems, navigation and signage'.

Where available, outcomes are supplemented with further research evidence. Given that the Thomson Reuters Web of Knowledge $^{\mathrm{SM}}$ has the largest database of journals, this was chosen as the vehicle for searches over the last 6 years, conducted using search terms such as 'blind' and 'visually impaired' in conjunction with terms of urban form such as 'kerb', 'pavement', 'sidewalk' and 'pedestrian' to locate studies.

\section{Key themes}

\subsection{Pedestrian priority}

The 'Streets ahead' campaign by Guide Dogs UK states that priority for pedestrians should take precedence over all other forms of transport on designated footways in traditional streets; and also in shared space designs. In comparison, such a pedestrian priority is less strongly worded in the Who 'Age friendly' cities report (Who, 2007) with the focus being on 'pedestrian-friendly' walkways, 'drivers giving way to pedestrians' and 'pedestrians having priority on pavements' with 'cycle paths ... separate from pedestrian walkways'.

Regarding the DfT local transport note (DfT, 2011), the shared space concept refers to 'reducing the dominance of motor vehicles and enabling all users to share the space' (on p. 6). These different stances regarding the priority of the pedestrian will clearly have their influence on the review that follows concerning walkways and pedestrian routes (including obstructions); pavements, kerbs and tactile paving; street crossing and intersections, obstructions; and access to public transit. Two general areas for future concern are first that the DfT local transport note (DfT, 2011) does not appear to address the implications around the uptake of hybrid electric vehicles, which are less easy to detect by blind pedestrians (Wall Emerson et al., 2011). Second, it does not address factors relating to the degree of unfamiliarity of space, which are issues for tourists/visitors who are blind and partially sighted.

\subsection{Walkways and pedestrian routes}

The Who 'Age friendly' cities report (Who, 2007) refers to key characteristics such as pedestrian walkways being 'accessible' and free from obstructions and 'having a smooth surface' and having public toilets on hand. Guide Dogs UK make explicit statements about the need to have clearly defined and obstaclefree pedestrian routes associated with a 'logical layout'. They refer to all streets - including those with shared space schemes as needing to 'include clear, continuous and readily identifiable routes that are clearly delineated from those used by cyclists and motorists'. Further, in the context of a shared space scheme, pedestrian routes between 'safe spaces' and footways in a traditional street should be 'continuous and unambiguous'.

Further, it is stated that in pedestrianised zones and open spaces reference points should be provided to assist blind and partially sighted people. Often this is achieved through different types of 'delineation'. Three particular approaches to achieving delineation are physical delineation (e.g. kerb, unless at a crossing point when it needs to be dropped), surface differentiation (e.g. tactile guidance paths) or visual contrast (e.g. a band and/or changes of colour) at surface level. This latter point is emphasised by the DfT local transport note (DfT, 2011), where it is pointed out that tonal (colour) contrast enables partially sighted users to perceive boundaries such as the edge of the carriageway. However, this report also acknowledges that complicated surface patterns can lead to disorientation.

Investigating this issue, Jenness and Singer (2008) studied 50 adults (aged 24-92 years) with some remaining useful vision but with limited visual acuity or visual field, and showed that detectable warning colours contrasting with pavement colour by a minimum luminance contrast of $60 \%$ could be seen from a 
distance of $2.44 \mathrm{~m}$ by around $92 \%$ of pedestrians under daylight conditions. The recommendation was that on dark pavements (e.g. asphalt) lighter coloured detectable warnings with a high reflectance index needed to be used to offer 'lighton-dark' contrast as opposed to 'dark-on-light' contrast.

Given that one hallmark of the shared space schemes is 'level' surfaces, it has been acknowledged that 'level surfaces, especially in busier settings, can create significant problems for blind and partially sighted people who often use kerbs to define comfort space and to navigate by. Where a level surface is desirable therefore, it may be necessary to implement mitigating measures' (DfT, 2011: p. 40) The DfT local transport note (DfT, 2011) described forms of appropriate demarcation with flexibility 'dependent on circumstance', citing as examples tactile paving, bollards or other street furniture. Demarcation information from bollards and street furniture is inevitably discontinuous. For pedestrians who are registered blind with little functional vision, this information is not continuously accessible and may be encountered as an obstruction rather than a navigation aid or demarcation line. Respective statements about obstructions to pedestrian flow are presented in Table 1. Although all three initiatives underscore the importance of obstruction-free mobility, the DfT local transport note (DfT, 2011) cites bollards as potential demarcation devices, whereas Guide Dogs UK indicates that bollards and other furniture (e.g. seats, planters etc.) need to be out of the main pedestrian flow and their position identifiable to all users. Additional demarcators like tactile paving and visual contrast could be used to aid in this endeavour. These specific features of demarcation are now addressed in more detail.

\subsection{Pavements, kerbs and surface differentiation}

In addition to the key features already outlined, Who agefriendly cities report (Who, 2007) also specifies that pavements should be non-slip, wide enough for wheelchairs and have dropped kerbs to road level to take into account users with mobility needs. The 'Streets ahead' campaign by Guide Dogs UK identifies kerbs and building lines as being critical reference points, especially for those using mobility devices such as guide dogs and long canes. Finally, the DfT local transport note (DfT, 2011) refers to the scope for a kerb-free design in shared space schemes and the role played by 'mitigating' measures as already discussed.

In the UK context, the DfT (2007) guidance on tactile paving schemes indicates that artificial surfaces can be utilised to create detectable safety warnings (e.g. blister surface) of carriageway edges, act as hazard alerts (e.g. corduroy surface) for top/bottom steps and guidance (e.g. rib and sinusoidal) to indicate routes.

Regarding the detectability of warning surfaces for blind pedestrians, a study by Stahl et al. (2010) compared a range of physical parameters. The study involved 12 participating blind pedestrians who were experienced at navigating pedestrian environments using a long white cane. The work showed that the chance of detecting a tactile warning surface did not depend on the existence of a kerb - and was unaffected by whether the warning surface was $1500 \mathrm{~mm}$ or $1000 \mathrm{~mm}$ in depth. Instead, the key factors were the structure of the surface, together with the 'distinct' (e.g. gravel to asphalt or gravel to grass) natural guidance surface prior to the warning

Source

Key statements

Who (2007)

Local transport note 1/11 (DfT, 2011)

Guide Dogs UK (2012)
'Pavements are well-maintained, free of obstructions and reserved for pedestrians.' 'Evidence suggests that the most important navigation feature for blind and partially sighted people is the building line, and this is best kept uncluttered by temporary obstructions such as A-boards. Temporary obstructions present a particular problem, as their locations cannot be 'learned'.'

'Obstacles that are temporary or not consistent in their use or location, for example advertising A-boards, cafe furniture and wheelie bins can be a problem for all users, but especially for blind and partially sighted people. Management and enforcement practices should be in place to ensure that all potential obstacles are either positioned in clearly defined ways away from pedestrian routes or removed.'

'All pedestrian circulation routes, whether in a traditional street or as a part of a shared space scene, should be free from obstacles. Where potential obstacles are incorporated, for example, seating, waste bins, bollards, planters, posts, signs and trees, these should be carefully designed and sited out of the main pedestrian flow and their position should be clearly identifiable to all users, including blind and partially sighted people, by the use of visual contrast and surface level tactile indicators and/or tapping rails.' 
Accessibility of urban spaces for visually impaired pedestrians Norgate surface. Under these test conditions, tactile warning surfaces with flat-topped domes with a minimum depth of $1000 \mathrm{~mm}$ were detected by experienced users with a white cane. Noticeably, Japanese and Swedish surfaces with flat-topped domes were more detectable than Danish or English surfaces. However, caution is needed in the interpretation of these results. The concentration on Swedish pedestrians potentially biases these results because of their familiarity with flat-topped domes. Also, the relatively small sample of white cane users did not necessarily represent the entire range of skills in this group.

It can also be asked whether tactile paving has any adverse impact for other users. The extent to which a single type of tactile surface impacted on sighted adults' gait was investigated in laboratory conditions, using $25 \mathrm{~mm}$ diameter and $5 \mathrm{~mm}$ high tactile blisters with adults aged over 60 years (Thies et al., 2011). The authors reported that rhythmic gait did become more variable, signifying a degree of change in balance. However, no falls were reported. These minor effects, along with the increase in prevalence of adults who are blind or partially sighted, would suggest that it is important to use a tactile paving, while minimising any adverse impact for sighted pedestrians.

Regarding older adult pedestrian injuries, data extracted from the US national electronic injury surveillance system-all injury programme indicated that nearly a quarter of pedestrians injured by a fall reported that a kerb was involved, and that adults aged over 80 years were more likely than those aged 6569 years to have a fall involving a kerb (Naumann et al., 2011). Painting or marking kerbs was cited as one solution (Naumann et al., 2011).

Kerb height is an important factor regarding whether or not kerbs are detected. Research reported by Thomas (2011) arising from the pedestrian accessibility movement and environmental laboratory in University College London, UK showed that when both bullnose and chamfer kerbs, with heights between $20 \mathrm{~mm}$ and up to $120 \mathrm{~mm}$, were tested with 36 participants, no-one failed to detect kerbs higher than $60 \mathrm{~mm}$. In contrast, kerbs of lower height were not always detected. Used in conjunction with dropped kerbs and tactile paving, a minimum kerb height was viewed as a way to delineate the walkway for all pedestrians, especially vulnerable users.

\subsection{Street crossings and intersections}

Unsurprisingly, the challenge of crossing a road safely raises particular issues, especially for blind pedestrians, as there tends to be reliance on unpredictable external sounds, such as car brakes, as cues regarding when to cross and when not to cross the road. In addition, there is the task of identifying a controlled crossing or an apparently suitable place to cross. Then comes the task of both adopting and maintaining an appropriate alignment of the crossing path. There is a tendency for blind pedestrians to veer 'off course' (Scott et al., 2011). Take the case of a pedestrian walking across the centre of a pedestrian crossing over four lanes of traffic (approximately 50 feet $(15 \cdot 24 \mathrm{~m})$ long). Assuming no veering or correction to the path - with $4^{\circ}, 6^{\circ}, 8^{\circ}$ and $10.5^{\circ}$ of initial misalignment they would deviate some $3 \cdot 5,5 \cdot 25,7$ or $9 \cdot 25$ feet (i.e. $1 \cdot 07,1 \cdot 60$, 2.13 and $2.82 \mathrm{~m}$ ) respectively from the centre line of the crossing. This means that with only $4^{\circ}$ of misalignment they would not finish crossing within the pedestrianised area. In a simulation study determining which cues, if any, facilitated alignment, the implementation of underfoot bars raised perpendicular to the desired walking direction aided staying 'on course' (Scott et al., 2011).

In the Who 'Age-friendly cities' initiative (Who, 2007), the emphasis is on drivers giving way to pedestrians at intersections and pedestrian crossings, and on a sufficient number of pedestrian crossings with 'non-slip' markings, visual and audio cues and appropriate crossing times. Such features are taken considerably further by the Guide Dogs UK 'Streets ahead' campaign. Their position is that controlled crossings should be prioritised over informal ones, and they specifically state that informal crossings should not replace controlled ones. Furthermore, they are more specific about the siting of these crossings, stating that controlled crossings need to be sited at the beginning and end of pedestrian zones and shared spaces. In addition, in the case of large areas or long streets, crossings need to be placed at key points where people need to cross. Guide Dogs UK also state that dropped kerbs and tactile paving should be provided at regular intervals, and particularly near bus stops and key destination points. Unlike Who (2007), Guide Dogs UK refer to the importance of providing information in dual modalities from audible and tactile signals (i.e. rotating cone) to enable safe crossings.

\subsection{Access to transit systems, navigation and signage}

The availability of accessible and affordable public transport is regarded by Who (2007) as a fundamental enabler for participation in society. Regarding accessibility, main concerns include 'Transport stops and stations are conveniently located, accessible, safe, clean, well-lit and well-marked, with adequate seating and shelter' as well as 'Complete and accessible information provided to users about routes, schedules and special needs facilities'.

As pointed out by Strickfaden and Devlieger (2011), little work to date has looked at how metro systems have been adapted for users with visual impairment. In transit stations there are often multiple access areas, ambiguous soundscapes and crowds, which reduce the chance of identifying landmarks and routes. A study by Gallagher et al. (2011) involved focus groups of 
blind or partially sighted participants from urban and rural areas in Ireland. The focus group reporting on experiences with buses showed that they often had to find their own way to where the bus has stopped, which may involve the hazard of stepping out into the road itself.

Regarding signage in streets, buildings and public transport venues, Who (2007) leaves the issue of what constitutes 'adequate' signage open to interpretation. Guide Dogs UK refers to the use of talking signs and emerging technologies as additional supplementary signage, and highlights the priority of promoting logical layout with consistent reference points. To this end, Marin-Lamellett and Aymond (2008) trialled an infra-red verbal guidance system in Auber Metro station, Paris, together with a tactile guidance system, with 32 blind or partially sighted users. The time to complete the trip was quicker with the two devices used together, and participants reported satisfaction with the kit.

\section{Conclusion}

Following the review of five themes relating to issues concerning the accessibility of urban areas for blind and partially sighted pedestrians, it is apparent from the content of three key sources (DfT, 2011; Guide Dogs UK, 2012; Who, 2007) that their statements are not entirely aligned. In particular, the Guide Dogs UK 'Streets ahead' campaign has pulled the pedestrian into the foreground and has devised a number of principles and interventions which can promote the safe and independent mobility of blind and partially sighted pedestrians. One key outcome here is that it is insufficient to plan and make provision for municipal services and facilities based exclusively on the needs of older adults without systematically also addressing the corresponding needs of a sub-group who are either blind or partially sighted. Although, on the face of it, the UK Equality Act (2010) would appear to enforce this general stance, it is the interpretation of the Act at 'street level' which needs considerable input. For instance, one concern is that there is risk for substantive underestimation of the environmental barriers that remain - for example, consider scenarios where blind or partially sighted pedestrians wish to visit an unfamiliar city which happens to adopt a combination of shared space and 'traditional' segregated areas. Consultations about street design between relevant parties (e.g. urban designers, street planners and authorities) and people with visual impairment need to examine the accessibility of urban spaces from the perspective of both local pedestrians and tourists. In order to achieve this, people with visual impairment and/or any key advocates need to be involved in all stages of design and delivery of street design. Even once the implementation phase is complete, and even on occasions when street environments are subsequently promoted as examples of agreed 'good practice', nevertheless there can remain both physical and social barriers experienced by people with visual impairment, and there needs to be a clear management strategy to ensure enforcement of practices for both these and other vulnerable road users.

In the future, with any substantial uptake of hybrid electric vehicles, there will be new risks to consider for shared spaces, especially for people who place reliance on sequential soundscapes of traffic noise to aid their alignment to cross roads.

Finally, with the emergence of a new generation of smart phone applications, there is scope to devise innovative personalised transport options for all adult pedestrians, including those who are blind and partially sighted. One example currently in 'proof of concept' stage is Sixth Sense Transport (see http://www.sixthsensetransport.com/) where the aim is to revolutionise the process of decision making in travel behaviour by using social networking principles to create 'visibility' and therefore 'accessibility' of potential transport options in both time and space.

Even on occasions when street environments are promoted as examples of 'good practice', nevertheless there can remain both physical and social barriers experienced by people with visual impairment, and there needs to be a clear management strategy to ensure enforcement of practices for both these and other vulnerable road users.

\section{REFERENCES}

Access Economics (2009) Future Sight Loss UK (1): The Economic Impact of Partial Sight and Blindness in the UK Adult Population. Access Economics Pty Ltd., London, UK, report for RNIB.

Alliance for Aging Research (1999) Independence for Older Americans: An Investment for our Nation's Future. Alliance for Aging Research, Washington D.C., USA.

Carroll J and Bentzen BL (1999) American Council of the Blind survey of intersection accessibility. The Braille Forum 38(7): $11-15$.

DfT (Department for Transport) (2007) Guidance on the Use of Tactile Paving Surfaces. DfT, London, UK. See http:// www.dft.gov.uk/publications/guidance-on-the-use-oftactile-paving-surfaces/ (accessed 28/09/2012).

DfT (2011) Local Transport Note 1/11 - Shared Space. DfT, London, UK.

Douglas G, Pavey S, Corcoran C and Clements B (2011) Evaluating the use of the ICF as a framework for interviewing people with a visual impairment about their mobility and travel. The British Journal of Visual Impairment 30(1): 6-21. See http://dx.doi.org/10.1177/ 0264619611428932 (accessed 05/11/2012).

Equality Act 2010 (2010) Elizabeth II. Chapter 15. The Stationery Office, London, UK.

Gallagher BAM, Hart PM, O'Brien C, Stevenson MR and Jackson AJ 
Accessibility of urban spaces for visually impaired pedestrians Norgate
(2011) Mobility and access to transport issues as experienced by people with vision impairment living in urban and rural Ireland. Disability and Rehabilitation 33(12): 979-988. See http://dx.doi.org/10.3109/09638288.2010.516786 (accessed 05/11/2012).

Guide Dogs UK (2012) Streets Ahead. Guide Dogs for the Blind Association, Reading, UK, campaign information. See http://www.guidedogs.org.uk/supportus/campaigns/streetsahead/ (accessed 15/03/2012).

Hamilton-Baillie B (2008) Towards shared space. Urban Design International 13(2): 130-138.

Jenness JW and Singer J (2008) Visibility and conspicuity of detectable warnings for pedestrians with visual impairments. Transportation Research Record 2073: 104-113. See http:// dx.doi.org/10.3141/2073-12 (accessed 05/11/2012).

Johnson V and Petrie H (1998) Travelling safely: the problems and concerns of blind pedestrians. The British Journal of Visual Impairment 16(1): 27-31.

Kaparias I, Bell MGH, Miri A, Chan C and Mount B (2012) Analysing the perceptions of pedestrians and drivers to shared space. Transportation Research Part F 15: 297-310.

Legood R, Scuffham P and Cryer C (2002) Are we blind to injuries in the visually impaired? A review of the literature. Injury Prevention 8(2): 155-160.

Marin-Lamellet C and Aymond P (2008) Combining verbal information and a tactile guidance surface: the most efficient way to guide people with visual impairment in transport stations. The British Journal of Visual Impairment 26(1): 63-81. See http://dx.doi.org/10.1177/ 0264619607083832 (accessed 05/11/2012).

MVA Consultancy (2009) Stage 1: Appraisal of Shared Space. DfT, London, UK.

MVA Consultancy (2011a) Shared Space: Operational Assessment. DfT, London, UK.

MVA Consultancy (2011b) Shared Space: Qualitative Research. DfT, London, UK.

Naumann RB, Dellinger AM, Haileyesus T and Ryan GW (2011) Older adult pedestrian injuries in the United States: causes and contributing circumstances. International Journal of Injury Control and Safety Promotion 18(1): 65-73. See http://dx.doi.org/10.1080/17457300.2010.517321 (accessed 05/11/2012).

Plouffe L and Kalache A (2010) Towards global age-friendly cities: determining urban features that promote active aging. Journal of Urban Health: Bulletin of the New York Academy of Medicine 87(5): 733-739. See http://dx.doi.org/ 10.1007/s11524-010-9466-0 (accessed 05/11/2012).

Scott AC, Barlow JM, Guth DA et al. (2011) Nonvisual cues for aligning to cross streets. Journal of Visual Impairment and Blindness October-November: 648-661.

Stahl A, Newman E, Dahlin-Ivanoff S, Almen M and Iwarsson S (2010) Detection of warning surfaces in pedestrian environments: The importance for blind people of kerbs, depth, and structure of tactile surfaces. Disability and Rehabilitation 32(6): 469-482. See http://dx.doi.org/10. 3109/09638280903171543 (accessed 05/11/2012).

Strickfaden M and Devlieger P (2011) The Brussels Metro: Accessibility through collaboration. Journal of Visual Impairment and Blindness October-November: 638-647.

Thies SB, Kenney LPJ, Howard D et al. (2011) Biomechanics for inclusive urban design: Effects of tactile paving on older adults' gait when crossing the street. Journal of Biomechanics 44(8): 1599-1604. See http://dx.doi.org/10. 1016/j.jbiomech.2010.12.016 (accessed 05/11/2012).

Thomas C (2008) Discussion: Shared space-safe space? Proceedings of the Institution of Civil Engineers - Municipal Engineeer 161(1): 59-60. See http://dx.doi.org/10.1680/ muen.2008.161.1.59 (accessed 05/11/2012).

Thomas C (2011) Briefing: Minimum effective kerb height for blind and partially sighted people. Proceedings of the Institution of Civil Engineers - Municipal Engineer 164(1): 11-13. See http://dx.doi.org/10.1680/muen.1000005 (accessed 05/11/2012).

Wall Emerson R, Naghshineh K, Hapeman J and Wiener W (2011) A pilot study of pedestrians with visual impairments detecting traffic gaps and surges containing hybrid vehicles. Transportation Research Part F 14(2): 117-127. See http:// dx.doi.org/10.1016/j.trf.2010.11.007 (accessed 05/11/2012). WHO (World Health Organisation) (2007) Global Age-Friendly Cities: A Guide. WHO, France.

\section{WHAT DO YOU THINK?}

To discuss this paper, please email up to 500 words to the editor at journals@ice.org.uk. Your contribution will be forwarded to the author(s) for a reply and, if considered appropriate by the editorial panel, will be published as discussion in a future issue of the journal.

Proceedings journals rely entirely on contributions sent in by civil engineering professionals, academics and students. Papers should be 2000-5000 words long (briefing papers should be 1000-2000 words long), with adequate illustrations and references. You can submit your paper online via www.icevirtuallibrary.com/content/journals, where you will also find detailed author guidelines. 